\title{
A chitosan-mediated inhalable nanovaccine against SARS-CoV-2
}

\author{
Shao-Hua Zhuo ${ }^{1, \S}$, Jun-Jun Wu ${ }^{1, \S}$, Lang Zhao ${ }^{1}$, Wen-Hao Li', Yu-Fen Zhao ${ }^{1,4}$, and Yan-Mei Li ${ }^{1,2,3}(\bowtie)$ \\ ${ }^{1}$ Key Lab of Bioorganic Phosphorus Chemistry \& Chemical Biology, Department of Chemistry, Tsinghua University, Beijing 100084, China \\ ${ }^{2}$ Beijing Institute for Brain Disorders, Beijing 100069, China \\ ${ }^{3}$ Center for Synthetic and Systems Biology, Tsinghua University, Beijing 100084, China \\ ${ }^{4}$ Institute of Drug Discovery Technology, Ningbo University, Ningbo 315221, China \\ ${ }^{\S}$ Shao-Hua Zhuo and Jun-Jun Wu contributed equally to this work.
}

(c) Tsinghua University Press and Springer-Verlag GmbH Germany, part of Springer Nature 2021

Received: 20 October 2021 / Revised: 19 November 2021 / Accepted: 22 November 2021

\begin{abstract}
Severe acute respiratory syndrome coronavirus 2 (SARS-CoV-2), with several antigenic variants, has grown into a global challenge, and the rapid establishment of an immune barrier is crucial to achieving long-term control of the virus. This has led to a great demand for easy preparation and scalable vaccines, especially in low-income countries. Here, we present an inhalable nanovaccine comprising chitosan and SARS-CoV-2 spike protein. The chitosan-mediated nanovaccine enabled a strong spikespecific antibody immune response and augmented local mucosal immunity in bronchoalveolar lavage and lungs, which might be capable of protecting the host from infection without systemic toxicity. In addition, the enhanced adaptive immunity stimulated by chitosan showed potential protection against SARS-CoV-2. Furthermore, inhalation of the nanovaccine induced a comparable antibody response compared to intramuscular injection. This inhalable nanovaccine against SARS-CoV-2 offers a convenient and compliant strategy to reduce the use of needles and the need for medical staff.
\end{abstract}

\section{KEYWORDS}

severe acute respiratory syndrome coronavirus 2 (SASR-CoV-2), inhalation, nanovaccine, chitosan

\section{Introduction}

Severe acute respiratory syndrome coronavirus 2 (SARS-CoV-2) has caused over 214 million cases and 4 million deaths as of August 26th, 2021, leading to the coronavirus disease 2019 (COVID-19) pandemic (https://coronavirus.jhu.edu/). The COVID-19 pandemic has urged the global community to develop and deploy therapeutics and vaccines rapidly to end the pandemic. However, the emergence of SARS-CoV-2 variants, such as B.1.1.7 (alpha) and B.1.617.2 (delta), has revealed altered virus infectivity and transmissibility [1-3], especially reduced neutralization [4-7], which possibly compromises vaccination, making it difficult to control the virus and the potential that it will coexist for many years. This highlights the need to establish herd immunity as soon as possible, leading to an increased demand for vaccines. Therefore, a pulmonary inhalable vaccine with simple preparation, convenient storage conditions, and the capacity for home vaccination can be an effective strategy to end the SARS-CoV-2 pandemic and prevent its return.

SARS-CoV-2 binds to the cell-surface receptor angiotensinconverting enzyme 2 (ACE2) by the viral spike (S) protein and then enters the host $[8,9]$. The respiratory tract is the primary route of virus invasion, including the nose and other mucosae, which are particularly susceptible to infection $[10,11]$. Therefore, respiratory tract mucosa-mediated immunity is critical for inducing effective and direct protection against SARS-CoV-2 [10]. Recently, some mucosal-related vaccines [12] were reported to induce strong antigen-specific IgG and secretory IgA antibody responses with great potential against SARS-CoV-2. However, most licensed vaccines are mainly administered by intramuscular injection [13-15], with limited mucosal immune responses. In contrast, pulmonary inhalation administration [16-18] outperformed the traditional injection method $[19,20]$, inducing relatively robust mucosal immunity and considerable antibody titers in some mucosal vaccines that block virus transmission or infection compared to intramuscular injection. In addition, a noninvasive and convenient inhalable vaccine enables household use, reduction of needle use and the need for large numbers of professional medical staff, which may allow it to be more easily accepted by the public to achieve global vaccination [21].

Mucoadhesives are crucial to prolonging antigen retention and tissue penetration, thus reducing the antigen dose. Chitosan (CS), with positive charges, is a natural mucoadhesive material with cGAS-STING-dependent adjuvanticity [22-24]. Many studies have indicated that chitosan is an effective mucosal vaccine adjuvant for many antigens [25-27], such as ovalbumin protein (OVA) [25] and receptor-binding domain (RBD) of SARS-CoV-2 spike protein [27]. Additionally, chitosan can open intercellular tight junctions temporarily [28], which is beneficial for drugs to pass through the intercellular space, thus improving tissue penetration and amplifying humoral and cellular immunity. Therefore, chitosan would be a candidate as an effective and safe mucosal adjuvant.

Inspired by the characteristics of chitosan and the negatively charged spike protein [29-31], we developed a chitosan-mediated pulmonary inhalable nanovaccine against the SARS-CoV-2 virus. 
The SARS-CoV-2 spike protein@chitosan (S@CS) nanovaccine was formed by physically mixing the SARS-CoV-2 spike protein and chitosan in one step. We observed the enhanced immunogenicity of the spike protein with the administration of chitosan nanovaccines, which induced SARS-CoV-2 spike-specific IgG antibody in sera and mucosal secretory $\operatorname{IgA}$ in bronchoalveolar lavage fluids (BALF). In addition to the systemic humoral response, the vaccine also augmented mucosal-mediated immunity in BALF and lungs, especially the $\mathrm{CD} 4^{+} \mathrm{T}$ cell-mediated Th2 immune response. Moreover, compared to intramuscular injection, the inhalable vaccine strategy induced comparable spikespecific antigen titers, confirming that chitosan could serve as a favorable mucosal vaccine adjuvant with aerosol inhalation. Therefore, the chitosan-mediated inhalable S@CS nanovaccine might provide a powerful, facile, and universal vaccine strategy [32] against SARS-CoV-2 and other influenza viruses, which also invade through the respiratory tract.

\section{Experimental}

\subsection{Preparation of S@CS nanoparticles (NPs)}

The S@CS NPs were prepared by mixing the chitosan (CS) and spike protein (S) via electrostatic interactions. CS solution was dissolved in $1 \%(\mathrm{v} / \mathrm{v})$ acetic acid solution. And the $\mathrm{pH}$ of solution was adjusted to 5.0-5.5. S was dissolved in phosphate buffered saline (PBS) at a concentration of $0.2 \mathrm{mg} / \mathrm{mL}$. After mixing the CS and $\mathrm{S}$, the solution was shaken for $4 \mathrm{~h}$ at $25^{\circ} \mathrm{C}$ in metal bath. The morphology of the S@CS NPs (CS: $5 \mu \mathrm{g}$, S: $5 \mu \mathrm{g}$ ) was observed by transmission electron microscopy (TEM). The size and zeta potential of S@CS NPs were obtained by Malvern zetasizer.

\subsection{Dendritic cells (DCs) activation and antigen presentation in vitro}

Bone marrow-derived dendritic cells (BMDCs) were isolated and cultured as follows. Female C57BL/6 mice were sacrificed and sterilized by alcohol. The legs were isolated to obtain bone marrow. After treating with red blood lysis buffer, the cells were resuspended in Roswell Park Memorial Institute (RPMI)-1640 complete medium (with $20 \mathrm{ng} / \mathrm{mL}$ granulocyte-macrophage colony stimulating factor (GM-CSF)). The same volume of fresh medium, containing $20 \mathrm{ng} / \mathrm{mL}$ GM-CSF, was added to the medium on day 3 and half of the cultured medium was changed with fresh medium on day 6 . The cells were collected and planted on plates on day 7-9. For the evaluation of cell immune activation, BMDCs were planted on 24 -well plate $\left(10^{6}\right.$ cells/well $)$ with different concentrations of chitosan (chitosan: 2, 4, and $8 \mu \mathrm{g} / \mathrm{mL}$ ) treatment. After incubation for $24 \mathrm{~h}$, the cells were collected and stained with antigen presenting cell (APC)-conjugated anti-mouse CD11c, phycoerythrin (PE)-conjugated anti-mouse CD86, and fluorescein isothiocyanate (FITC)-conjugated anti-mouse CD40 (dilution 1:200). After 1-h incubation in ice, cells were washed and analyzed by CytoFlex LX flow cytometry. Besides, the cell culture supernatant was centrifuged for cytokines measurement by enzyme-linked immunosorbent assay (ELISA) kit.

DC2.4 cells were cultured in RPMI-1640 containing 10\% fetal bovine serum (FBS) and 1\% penicillin-streptomycin. The cells were collected and planted on 24-well plate with a density of $2 \times$ $10^{5}$ cells/well and cultured for $6 \mathrm{~h}$. Next, the medium was replaced with fresh RPMI-1640 complete medium (CS: $2 \mu \mathrm{g} / \mathrm{mL}$, OVA: 2 $\mu \mathrm{g} / \mathrm{mL}$ ). After incubation for $24 \mathrm{~h}$, the cells were collected, washed, and stained with PE-conjugated anti-mouse CD86, FITCconjugated anti-mouse $\mathrm{CD} 40$, and APC-conjugated anti-mouse $\mathrm{H}$ $2 \mathrm{~K}^{\mathrm{b}}$ bound to SIINFEKL antibody. Finally, the cells were resuspended in PBS and analyzed by flow cytometry.

\subsection{Cytotoxicity assay}

DC2.4 cells were cultured, collected, and planted with a density of $10^{4}$ cells/well and cultured for $6 \mathrm{~h}$. Next, the medium was replaced with different concentration of chitosan (chitosan: 0, 0.5, 1, 2, 4, 8, $10,20,50$, and $100 \mu \mathrm{g} / \mathrm{mL}$ ) treatment. After incubation for $24 \mathrm{~h}$, cell counting kit-8 $(10 \mu \mathrm{L} /$ well $)$ was added and cultured for another $4 \mathrm{~h}$. Finally, the value of OD450 was read by microplate reader (Synergy 4, Biotek).

\subsection{Nanovaccines immunization in vivo}

All animal experiments were performed according to the institutional guidelines for animal welfare and were approval by the Institutional Animal Care and Use Committee (IACUC) at Tsinghua University. The laboratory animal facility has been accredited by Association for Assessment and Accreditation of Laboratory Animal Care International (AAALAC). All mice were obtained and housed in a facility of the Center of Biomedical Analysis at Tsinghua University.

$\mathrm{BALB} / \mathrm{c}$ mice (6-8 weeks, female) inhaled with microsprayer aerosolizer (35 $\mu \mathrm{L} /$ mouse, BJ-PW-M, BioJane, China). For evaluation of the nanovaccines stimulation, mice were separated randomly into five groups, including PBS, free $\mathrm{S}$ ( $5 \mu \mathrm{g} / \mathrm{mouse}$ ), S@CS(M) (medium dose: chitosan: $50 \mu \mathrm{g} /$ mouse), S@CS(H) (high dose: chitosan: $100 \mu \mathrm{g} /$ mouse), and Alhydrogel ${ }^{\circledR}$ adjuvant $2 \%$ plus spike (Alum+S, Alum: $100 \mu \mathrm{g} /$ mouse, intramuscular injection, 60 $\mu \mathrm{L} /$ mouse). Mice were vaccinated three times biweekly. Sera were collected seven days after each immunization. The mouse weight was recorded on day $0,7,14,21$, and 28 . Spleen, BALF, and lungs were collected three days after the third immunization.

For evaluation of the different administration routes in immune activation, mice were separated into four groups. Three groups were inhaled, including the PBS group, S (5 $\mu \mathrm{g} /$ mouse) and S@CS (inhalation) (dose: chitosan: $5 \mu \mathrm{g} /$ mouse). S@CS was also intramuscularly injected as a control (S@CS i.m.). Mice were vaccinated three times biweekly. Sera were collected at indicated times.

\subsection{Tissue processing}

Spleen was grinded and filtrated with $40 \mu \mathrm{m}$ cell strainers. Next, the red blood lysis buffer was added and incubated for $7 \mathrm{~min}$ at room temperature, and terminated with an equal volume of PBS. After centrifuging and suspended with PBS or medium, single-cell suspensions were obtained for flow cytometry or IFN- $\gamma$ enzymelinked immune absorbent spot assay (IFN- $\gamma$ ELISPOT).

The lungs were cut into pieces and digested with collagenase IV $(2 \mathrm{mg} / \mathrm{mL})$ and DNase I $(5 \mathrm{mg} / \mathrm{mL})$ at $37^{\circ} \mathrm{C}$ for $60 \mathrm{~min}$. After $1 \mathrm{~h}$ of treatment, the lungs were grinded, filtrated with $40 \mu \mathrm{m}$ cell strainers and centrifuged at $1,700 \mathrm{rpm}$ for $5 \mathrm{~min}$. Then, the lymphocytes were isolated with $40 \%$ Percoll in PBS, centrifuged at 2,800 rpm for $15 \mathrm{~min}$. Finally, the red blood lysis buffer was added and centrifuged to obtain the single cell suspension for flow cytometry.

\subsection{BALF collection}

Mice were sacrificed to collect BALF. Firstly, mouse throat was exposed, and then a $25 \mathrm{G}$ retention needle was inserted and fixed with a rope. Next, $0.8 \mathrm{~mL}$ of D-hanks was injected and pumped back and forth three times. This process was repeated three times to collect all the lavage fluid. Finally, the BALF was centrifuged at $1,000 \mathrm{rpm}$ for $10 \mathrm{~min}$. The supernatant was collected for spikespecific antibodies and cytokines detection. Finally, the cells were resuspended for flow cytometry analysis. 


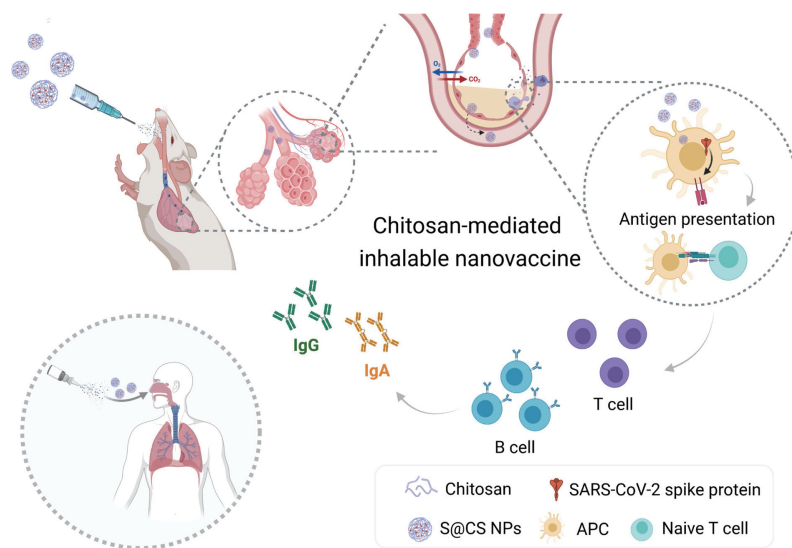

Scheme 1 Schematic illustration of the inhalable S@CS nanovaccine against SARS-CoV-2. The CS-mediated inhalable nanovaccine amplifies spike protein immunogenicity due to cGAS-STING-dependent adjuvanticity and the mucoadhesives of chitosan. Next, DC maturation, antigen presentation, and T cell activation contributed to a significant increase of SARS-CoV-2 spike-specific antibodies, which was critical for SARS-CoV-2 regression.

\subsection{ELISA}

SARS-CoV-2 spike-specific antibody titers, including IgG and IgA, was determined by ELISA. Spike protein $(1 \mu \mathrm{g} / \mathrm{mL}, 100 \mu \mathrm{L} /$ well $)$ was dissolved in $\mathrm{NaHCO}_{3}$ buffer ( $\mathrm{pH}$ 9.6) and pre-coated onto 96well ELISA plate (Costar 3590) at $4{ }^{\circ} \mathrm{C}$ overnight. Next, ELISA plate was washed and blocked by $0.25 \%$ gelatin PBS solution at room temperature. After $3 \mathrm{~h}$ of treatment, the well was washed with PBST solution (PBS buffer with $0.05 \%$ Tween) four times and the pre-diluted sera or BALF was added by $100 \mu \mathrm{L} /$ well for 1.5 $\mathrm{h}$ at $37^{\circ} \mathrm{C}$. Next, the well was washed and the rabbit anti-mouse IgG-Peroxidase antibodies (IgG-HRP: dilution 1:2,000) or the goat anti-mouse IgA-Peroxidase antibodies (IgA-HRP: dilution 1:10, 000) were added for another $1.5 \mathrm{~h}$ at $37^{\circ} \mathrm{C}$. Finally, the well was washed again and the 3,3',5,5'-tetramethylbenzidine (TMB) solution $(100 \mu \mathrm{L} /$ well $)$ was added for $1-5 \mathrm{~min}$ and terminated with $2 \mathrm{M} \mathrm{H}_{2} \mathrm{SO}_{4}(50 \mu \mathrm{L} /$ well). The value of $\mathrm{OD} 450$ was read by microplate reader (Synergy 4, Biotek).

SARS-CoV-2-specific antibody isotypes assay detecting IgG1, IgG2a, IgG2b, IgG3, IgA, and IgM, was similar to the procedures described above (S: sera dilution 1:200, S@CS nanovaccines: sera dilution 1:6,400, Alum+S: sera dilution 1:800). After incubation with sera for $1.5 \mathrm{~h}$, isotype antibodies were added respectively (dilution 1:1,000) and incubated for another $1.5 \mathrm{~h}$. Then, the well was washed and the rabbit anti-goat IgG-Peroxidase antibodies (IgG-HRP: dilution 1:1,000) were added for another $1.5 \mathrm{~h}$ at $37^{\circ} \mathrm{C}$. The following procedures were consistent with the described above. BALF cytokines were analyzed by mouse ELISA kits (DAKEWE), including interferon (IFN)- $\gamma$, tumor necrosis factor (TNF)- $\alpha$, interleukin (IL)-4, and IL-5 ELISA kits.

\subsection{Biosafety analysis in vivo}

For serum chemistry analysis, the sera were isolated three days after the third immunization. For evaluation of the tissue inflammation or organ damage assay, female $\mathrm{BALB} / \mathrm{c}$ mice were endotracheally administrated with $\mathrm{PBS}$, free spike protein, S@CS(M), and S@CS(H) (35 $\mu \mathrm{L} /$ mouse). One day after the first immunization, mouse heart, liver, spleen, lung, and kidney were collected and fixed in $4 \%$ paraformaldehyde (PFA) solution. Then, the tissues were cut into slices for hematoxylin and eosin (H\&E) staining assay after embedding in paraffin.

\section{Results and discussion}

\subsection{The immunostimulation of S@CS NPs}

The NPs were prepared by mixing the CS and the antigen together based on the electrostatic interactions, which were amenable to scale up. To confirm that CS and spike protein formed NPs successfully, transmission electron microscopy (TEM) imaging, and dynamic light scattering (DLS) were performed. As shown in Figs. 1(a) and 1(b), the images indicated the spherical morphology of the S@CS NPs, and the average diameter of the nanoparticles was approximately $210 \mathrm{~nm}$, which was efficient for mucosal binding [33]. Besides, the change of zeta potential after preparation confirmed the electrostatic interaction between CS and spike protein (Fig. 1(b)).

CS has been reported to be an adjuvant stimulating the cGASSTING pathway [22]. Therefore, CS, with a medium molecular weight of $190-310 \mathrm{kDa}$ and $75 \%-85 \%$ deacetylation, was evaluated in vitro to confirm its immunological activity. We evaluated the immune activation of bone marrow-derived dendritic cells (BMDCs) by CS after $24 \mathrm{~h}$ of incubation. The percentage of CD11c ${ }^{+}$DCs (Fig. S1(a) in the Electronic Supplementary Material (ESM)) was increased after CS treatment compared with the control group, indicating that CS could effectively stimulate DC maturation. The expression of activated $\mathrm{CD}^{+} 6^{+}$and $\mathrm{CD} 40^{+}$DCs (Figs. S1(b) and S1(c) in the ESM) was substantially upregulated in a dose-dependent manner, which was essential to stimulating prime $\mathrm{T}$ cells and amplify the immune response. Moreover, the $\mathrm{CD} 40^{+} \mathrm{CD} 86^{+}$DCs (Fig. 1(c) and Fig. $\mathrm{S} 1(\mathrm{~d})$ in the ESM) increased remarkably in the CS groups. ELISA kits were further used to investigate the secretion of cytokines by BMDCs after CS incubation. The secretions of CXCL10, IFN- $\beta$, IL6 , and TNF- $\alpha$ were increased significantly after CS treatment, as expected (Figs. 1(d)-1(g), Fig. S2 in the ESM). This result may be attributed to the adjuvanticity of CS, which triggers the STING pathway [22] to induce the secretion of type I interferons (IFNs), especially INF- $\beta$, and the chemokine CXCL10. The increased cytokine levels showed the ability to predict the presence of an early $\mathrm{CD}^{+} \mathrm{T}$ cell response in SARS-CoV-2 [34]. We also investigated the toxicity of CS on DC2.4 cells treated with different concentrations of CS using a cell counting kit-8 (CCK-8) assay. As shown in Fig. S3 in the ESM, CS had no effects on cell viability. Taken together, these results indicated that CS could trigger DC maturation and activation, which were important for antigen presentation and immune activation in vivo.

Next, to assess whether CS could promote antigen presentation on DCs in vitro, antigen-presenting DC2.4 cells were adopted. OVA, a model antigen, was selected for this section. As shown in Figs. 1(h) and 1(i), CS was able to induce DC2.4 cells activation with upregulation of CD40 and OVAp-major histocompatibility complex I complex (OVAp-MHC-I) expression compared with the free antigen group. It is worth noting that antigen recognition, processing, and presentation are indispensable for the sufficient activation of adaptive immunity, which is necessary for COVID19 convalescent patients with impaired and insufficient cellular immunity [35]. These data indicated that CS-mediated nanovaccines showed great potential in mucosal immunity against pulmonary disease based on augmented antigen presentation and retention in lungs [16], owing to the adjuvant effects and mucoadhesives of CS.

\subsection{Inhalable S@CS nanovaccine amplified systemic antibody responses}

The immunogenicity of spike protein was investigated in BALB/c mice, which were administered PBS, spike protein, S@CS(M) 
(a)

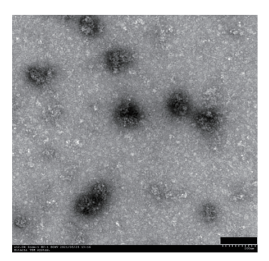

BMDC
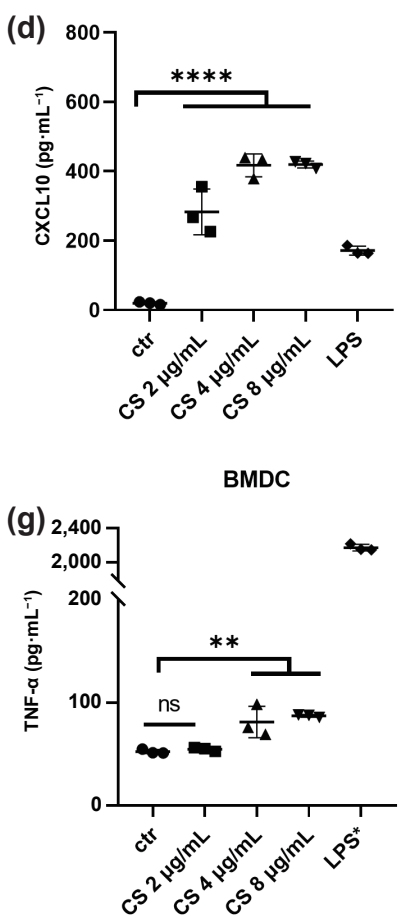

(b)

\begin{tabular}{|c|c|c|}
\hline & $\begin{array}{c}\text { Particle size } \\
(\mathrm{nm})\end{array}$ & $\begin{array}{c}\text { Zeta potential } \\
(\mathrm{mV})\end{array}$ \\
\hline S@CS & $210.3 \pm 2.1$ & $5.68 \pm 1.03$ \\
\hline
\end{tabular}

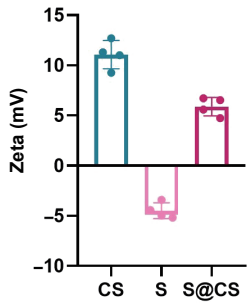

(e)

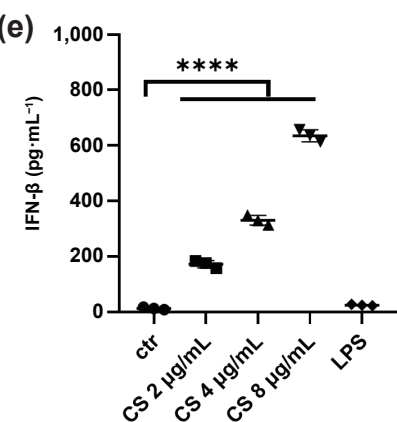

DC2.4

(h)

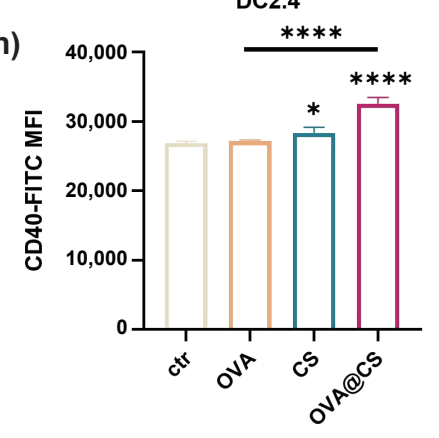

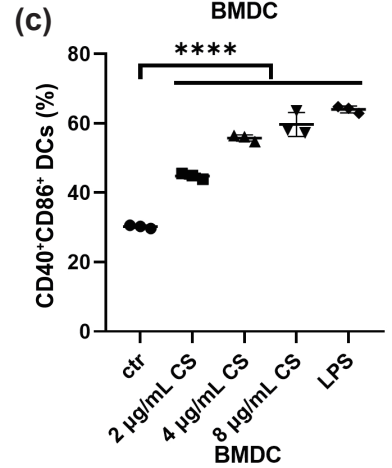

(f)

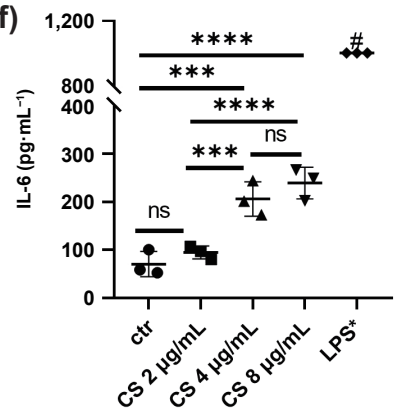

(i)

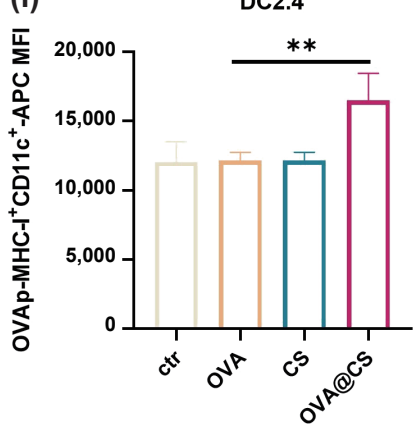

Figure 1 Immune activation analyses of CS-mediated nanovaccines. (a) The morphology of S@CS nanoparticles by TEM. Scale bar: 200 nm. (b) Zeta potential and particle size of the S@CS nanoparticles (polydispersity (PDI): $0.457 \pm 0.042$ ). (c)-(i) Evaluation of the immune activation of BMDCs by CS. The percentage of CD40 ${ }^{+} \mathrm{CD} 86^{+}$DCs (c) and the secretion of cytokines, including CXCL10 (d), IFN- $\beta$ (e), IL-6 (f), and TNF- $\alpha$ (g). \#: The value was out of range and artificially set to 1, 000. (h)-(i) Relative quantification of CD40 and the OVAp-MHC-I complex (CS: $2 \mu \mathrm{g} / \mathrm{mL})$ on DC2.4 cells. All data were presented as the mean \pm standard deviation (SD), and statistical analysis was performed by one-way ANOVA: ${ }^{\star} P<0.05,{ }^{\star *} P<0.01,{ }^{\star * \star} P<0.001,{ }^{\star * *} P<0.0001$. ns, no significant differences.

(medium dose, CS: $50 \mu \mathrm{g} /$ mouse) or S@CS(H) (high dose, CS: 100 $\mu \mathrm{g} / \mathrm{mouse})$ nanovaccine through pulmonary inhalation. Alum (100 $\mu \mathrm{g} /$ mouse) plus spike (Alum $+\mathrm{S})$ was intramuscularly injected. Sera were collected at the indicated times after immunization with different treatments (Fig. 2(a)). The SARSCoV-2 spike-specific IgG antibody titers in sera in individual animals were analyzed by ELISA. As shown in Figs. 2(b) and 2(c), the S@CS groups induced higher IgG antibody titers than the free spike group and alum group on day 7 after a single inhalation. While the level of IgG antibody titers in S@CS(M) was slightly higher than that in S@CS(H) on day 7, there were no significant differences between the medium-dose group and the high-dose group on day 21 , indicating that the S@CS(M) nanovaccine induced rapid antibody responses compared with the S@CS(H) nanovaccine. The results demonstrated that CS improved spike immunogenicity and exerted an efficient immune response. Then, the mice were boosted on day 14 , and sera were collected one week after the second vaccination. Surprisingly, as shown in Figs. 2(d) and 2(e), the spike-specific IgG antibody titers of the S@CS(M) group were elevated to approximately $5 \times 10^{4}$, which were 113.3- and 10.6-fold higher than those of the free spike group and Alum $+S$, respectively. These data indicated that CS could significantly amplify the SARS-CoV-2 spike-specific humoral response.
To further dissect the balance between type I-based immunity (Th1) and type II-based immunity (Th2), we assessed antibody isotypes, including IgG1, IgG2a, IgG2b, IgG3, IgM, and IgA, in sera on day 21 with corresponding dilutions. Generally, IgG1 is related to Th2 immunity, and IgG2a is correlated with Th1 immunity [36-38]. Therefore, the ratio of $\operatorname{IgG} 1 / \operatorname{IgG} 2$ a could reflect the relative contribution of Th1 and Th2 immunity [39-41]. As shown in Fig. 2(f), IgG1 was the primary antibody isotype, which reflected augmented humoral immunity. Notably, the ratio of IgG1/IgG2a was close to 2.4 in the S@CS nanovaccine groups, indicating that the inhalable nanovaccine generated an enhanced contribution of Th2 immunity. Moreover, the amount of IgG2a was dispensable for the activation of cellular immunity. The isotype assay identified a CS-mediated inhalable nanovaccine as a feasible strategy for stimulating effective humoral immunity, accompanied by moderate cellular immunity.

\subsection{Inhalable S@CS nanovaccine enhanced mucosal} antibody responses

Secretory $\operatorname{IgA}(\operatorname{sg} \mathrm{A})$, a mucosal antibody at the first line against respiratory pathogens, shows potential protection against SARS$\mathrm{CoV}$ infection $[38,39]$. Studies have reported that SARS-CoV-2specific IgA dominates with intranasal injection but not through 
(a)

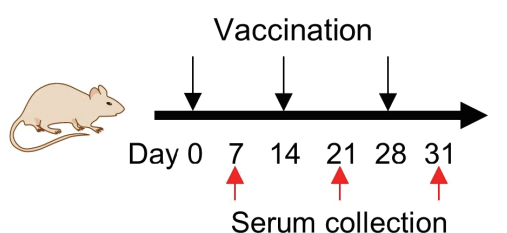

(b)

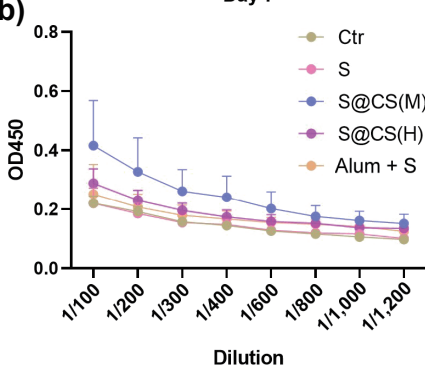

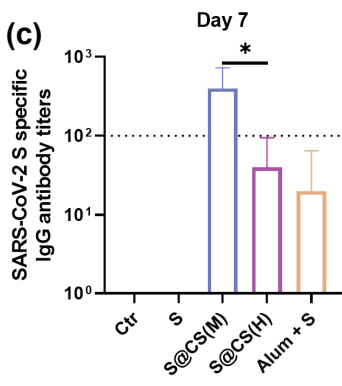
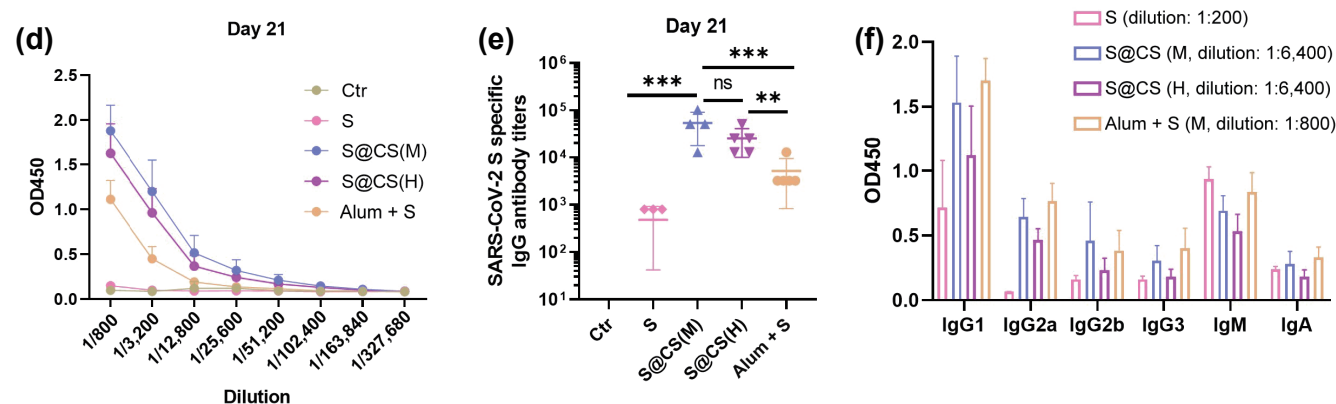

Figure 2 The S@CS nanovaccine enhanced the SARS-CoV-2 spike-specific antibody response in sera in vivo. (a) Schedule of immunization and serum collection in $\mathrm{BALB} / \mathrm{c}$ mice $(n=4-5)$. Mice were separated into five groups and underwent inhalation with a microsprayer aerosolizer, including the PBS group (ctr), $\mathrm{S}(5 \mu \mathrm{g} / \mathrm{mouse})$, S@CS(M) (medium dose: chitosan: $50 \mu \mathrm{g} / \mathrm{mouse}$ ), S@CS(H) (high dose: chitosan: $100 \mu \mathrm{g} / \mathrm{mouse}$ ), and Alum plus spike (Alum+S, Alum: $100 \mu \mathrm{g} / \mathrm{mouse}$, intramuscular injection). (b) and (c) SARS-CoV-2 spike-specific IgG antibody titers in sera on day 7 after a single immunization. (d) and (e) SARS-CoV-2 spike-specific IgG antibody titers in sera on day 21 after the second immunization. (f) The SARS-CoV-2 spike-specific antibody isotype on day 21, with corresponding dilutions (S: dilution: 1:200, S@CS nanovaccines: dilution: 1:6,400, Alum + S: dilution: 1:800). All data were presented as the mean \pm SD, and statistical analysis was performed by one-way ANOVA: ${ }^{\star} P<0.05,{ }^{* *} P<0.01,{ }^{* * *} P<0.001,{ }^{* * *} P<0.0001$. ns, no significant differences.

an intramuscular route [42-45], suggesting that IgA exerts protective mucosal immunity in pulmonary infection. Furthermore, IgA plays an important role in early SARS-CoV-2specific humoral responses $[43,44]$. Therefore, sIgA in bronchoalveolar lavage fluid (BALF) from immunized mice was analyzed by ELISA three days after the third immunization. The S@CS nanovaccine, but not the free spike protein, increased the spike-specific IgA response (Figs. 3(a) and 3(b)). The results demonstrated that the noninvasive pulmonary inhalable nanovaccine could be an efficient strategy to induce sufficient local mucosal immunity against SARS-CoV-2 infection, which is mainly due to the effect of adjuvants and the natural mucoadhesives of CS. In addition, SARS-CoV-2 spike-specific IgG antibody titers in BALF (Figs. 3(c) and 3(d)) were detected, which were consistent with the results in sera. Taken together, according to the spike-specific antibody titers in BALF and sera, the CSmediated vaccine could elevate not only the systemic response but also local mucosal immunity.

\subsection{Inhalable S@CS nanovaccine elicited spike-specific T cell responses in the spleen}

We sought to delineate factors contributing to humoral immunity with S@CS nanovaccine inhalation. Increasing data have revealed that the $\mathrm{T}$ cell immune response is critical for SARS-CoV-2 convalescent individuals [46-48]. Grifoni et al. [46] reported that a high frequency of SARS-CoV-2-specific T cells was identified in COVID-19 convalescent patients, with $70 \% \mathrm{CD}^{+} \mathrm{T}$ and $100 \%$ $\mathrm{CD} 4{ }^{+} \mathrm{T}$ cell. To investigate whether the S@CS nanovaccine could trigger the SARS-CoV-2 spike-specific $\mathrm{T}$ cell response, spleen samples were collected after the third immunization for IFN- $\gamma$ enzyme-linked immune absorbent spot (ELISPOT) assay and intracellular granzyme B cytokine staining assay after spike restimulation ex vivo. The spike-specific IFN- $\gamma^{+} \mathrm{T}$ cell response in the S@CS $(\mathrm{H})$ group increased to 24.2-fold higher than that in the free spike protein group, reaching a mean number of 425 spike-
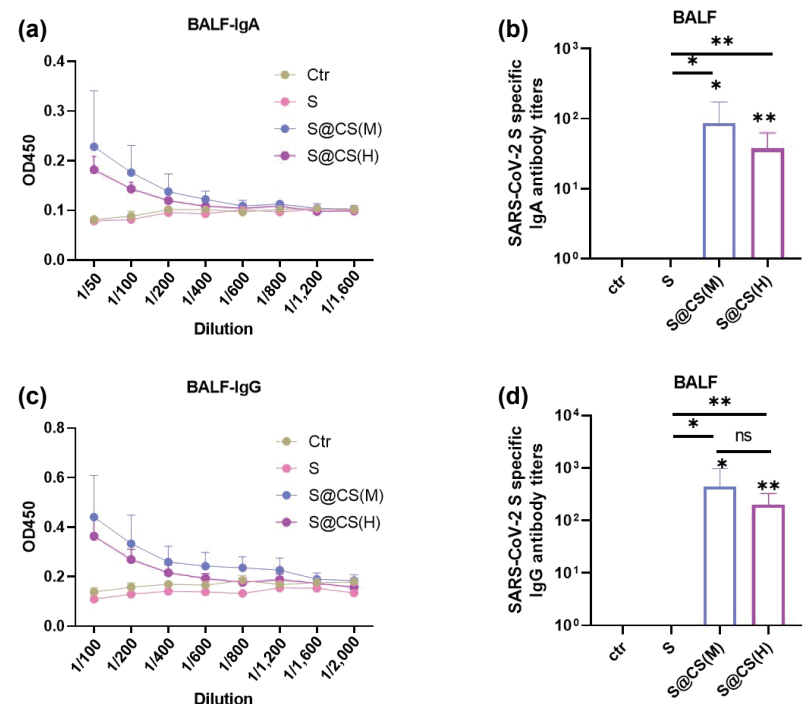

Figure3 The S@CS nanovaccine stimulated the SARS-CoV-2 spike-specific antibody response in BALF. (a) and (b) SARS-CoV-2 spike-specific IgA antibody titers in BALF on day 31 after the third immunization. (c) and (d) SARS-CoV-2 spike-specific IgG antibody titers in BALF on day 31 after the third immunization. All data were presented as the mean $\pm \mathrm{SD}$, and statistical analysis was performed by one-way ANOVA: ${ }^{*} P<0.05,{ }^{*} P<0.01$, ${ }^{* *} P<0.001$, ${ }_{* * * *} P<0.0001$. ns, no significant differences.

specific T cells per $10^{6}$ splenocytes (Fig. 4(a)). Compared to the control group and the free spike group, the ratio of antigenspecific granzyme $\mathrm{B}^{+} \mathrm{CD} 8^{+} \mathrm{T}$ cells in splenocytes (Fig. $\mathrm{S} 4$ in the ESM) was also increased significantly in the S@CS(H) group, suggesting powerful spike-specific cellular immunity for clearance of viral infections. This result might be attributed to the comprehensive adjuvanticity of CS and the natural properties of mucoadhesives, which prolong antigen retention and penetration through tight junctions. 
(a)
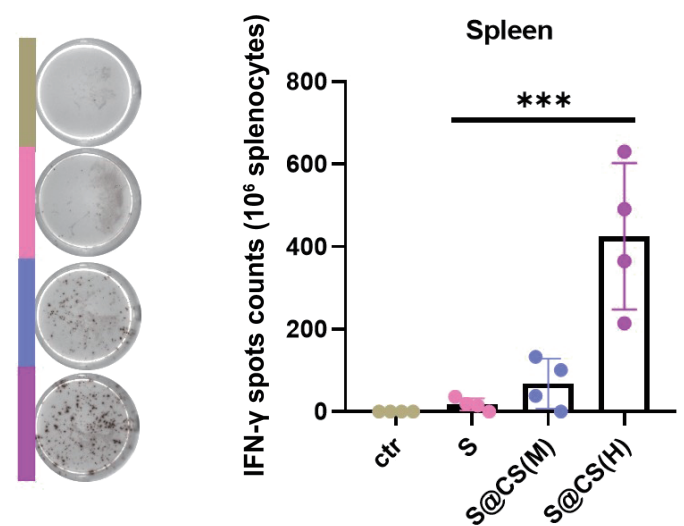

Spleen

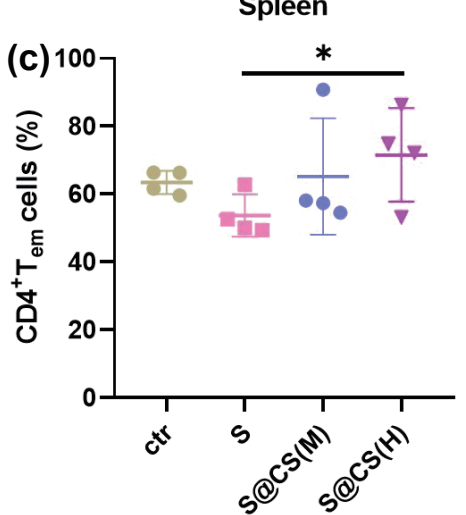

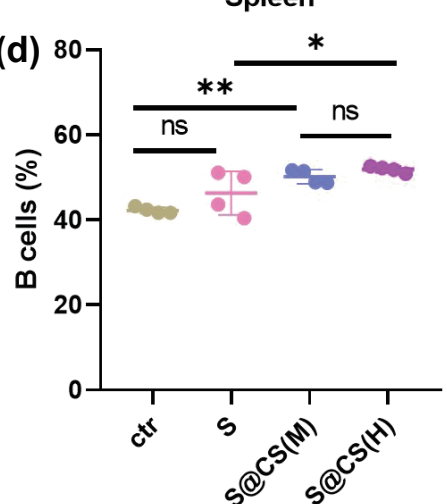

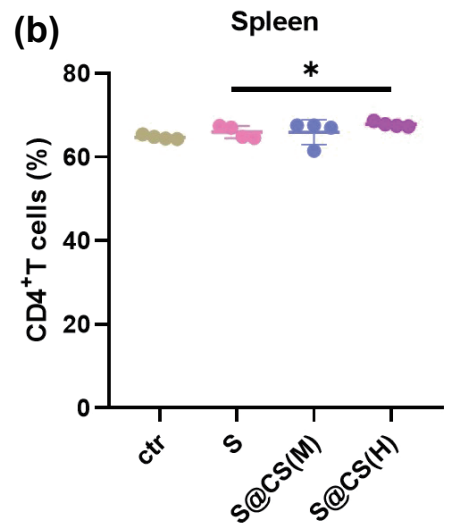

Spleen

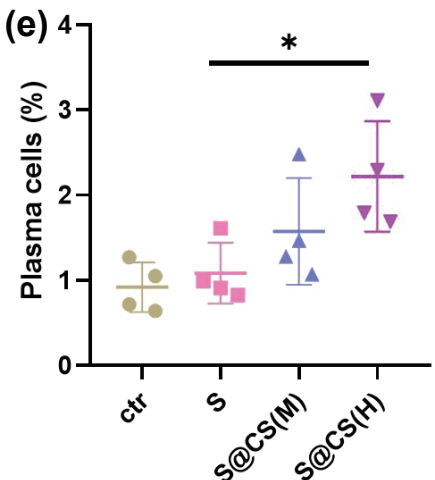

Figure 4 The S@CS nanovaccine induced the spike-specific T cell response in spleen after the third immunization. (a) Spike-specific IFN- $\gamma$ ELISPOT assay on splenocytes isolated on day 31. (b) and (c) Frequency of CD4 ${ }^{+} \mathrm{T}$ cells and $\mathrm{CD} 4^{+}$Tem cells (defined as CD3 ${ }^{+} \mathrm{CD} 4^{+} \mathrm{CD} 44^{+} \mathrm{CD} 62 \mathrm{~L}^{-}$) in spleen. (d) and (e) Frequency of B cells (defined as CD45R/B220 $\mathrm{B}$ cells) and plasma cells (defined as $\mathrm{CD} 45 \mathrm{R} / \mathrm{B} 220^{+} \mathrm{CD} 138^{+}$cells). All data were presented as the mean $\pm \mathrm{SD}$, and statistical analysis was performed by one-way ANOVA: ${ }^{*} P<0.05,{ }^{* *} P<0.01,{ }^{* * *} P<0.001,{ }^{* * *} P<0.0001$. ns, no significant differences.

Emerging data suggest that memory immunity [48], which may persist for a long time, is important for inducing long-term protection against SARS-CoV-2. Therefore, memory $\mathrm{T}$ cells in splenocytes were detected following the third immunization by flow cytometry. As shown in Figs. 4(b) and 4(c), S@CS nanovaccine treatment enhanced the percentage of $\mathrm{CD} 4^{+} \mathrm{T}$ cell and effector memory $\mathrm{T}$ cells (EM), defined as $\mathrm{CD}^{+}{ }^{+} \mathrm{CD} 4{ }^{+} \mathrm{CD} 44^{+} \mathrm{CD} 62 \mathrm{~L}^{-}$. The data demonstrated that $\mathrm{CD} 4{ }^{+} \mathrm{T}$ cells might play a crucial role in regulating humoral immunity and cellular immunity. Furthermore, $\mathrm{CD}^{+} \mathrm{T}$ cells are capable to instruct B cells and are relevant to spike-specific B cells in COVID$19[34,49]$. Therefore, B cells (defined as CD45R/B220+ cells) and plasma cells (defined as $\mathrm{CD} 45 \mathrm{R} / \mathrm{B} 220^{+} \mathrm{CD} 138^{+}$cells) were detected. As shown in Figs. 4(d) and 4(e), B cells and plasma cells were amplified with nanovaccine inhalation, which was beneficial for the production of antigen-specific antibodies. Taken together, S@CS nanovaccine inhalation was critical for inducing strong humoral immunity and spike-specific cellular immunity. However, the results of the medium-dose group and the high-dose group were slightly different from the antibody titers. This might be due to the renal targeting [50] and adjuvanticity of CS. While compared to the medium dose of CS, the high dose of CS tended to be metabolized more quickly by kidneys, CS activated immune cells in a dose-dependent manner.

\subsection{Inhalable S@CS nanovaccine augmented the mucosal immune response in BALF and lungs}

Previous studies $[8,9]$ have revealed that lungs are the most susceptible and vulnerable organs in the COVID-19 pandemic. In addition to the spike-specific antibody response and the specific $\mathrm{T}$ cell response in mice, we next analyzed innate and adaptive immune cells in BALF and lungs. The BALF samples and the lungs were isolated on day 31, three days following the third immunization. As expected, inhalable pulmonary vaccines could stimulate and recruit immune cells in lungs efficiently. We first examined DC cells, B cells, and T cells in lungs. As shown in Fig. S5(a) in the ESM, the amount of CD11c ${ }^{+}$DCs in lungs activated by the nanovaccine was higher than that activated by free spike. While the percentage of DCs in the medium-dose group was relatively weak, the activation of mature DCs, defined as CD86 ${ }^{+} \mathrm{CD} 11 \mathrm{c}^{+}$DCs (Fig. 5(a)), was 3.9 times higher than that in the free spike group. And the high-dose group was 7.4 times and 1.9 times that of the free spike group and the medium-dose group, respectively, indicating that the inhalable nanovaccine could prominently activate DCs and trigger mucosal immunity. Subsequently, $\mathrm{CD}_{103}{ }^{+} \mathrm{CD} 11 \mathrm{c}^{+}$DCs (CD103: an antigen crosspresentation marker [51]) were evaluated (Fig. 5(b)). In alignment with the results obtained from DC2.4 cells in vitro, the proportion of $\mathrm{CD}_{103} \mathrm{CD}^{+} 1 \mathrm{c}^{+}$DCs increased 2.0-fold in the medium-dose group and 4.8-fold in the high-dose group compared to the free spike group. The results revealed augmented antigen presentation by the S@CS nanovaccine, indicating that CS was crucial for antigen delivery and cross-presentation. In addition, the number of $\mathrm{F} 4 / 80^{+}$and $\mathrm{CD} 86^{+} \mathrm{F} 4 / 80^{+}$macrophage cells were enhanced significantly in S@CS nanovaccine groups in BALF (Figs. 5(f) and 5(g), Fig. 66 in the ESM). These data suggested that the S@CS nanovaccine substantially increased the levels of $\mathrm{T}$ cell recognition and activation. As shown in Figs. 5(c)-5(e), 5(h)-5(j), the inhalable nanovaccine stimulated $\mathrm{T}$ cell differentiation and activation in lungs and BALF, with an increased percentage of $\mathrm{CD}^{+}$cell, $\mathrm{CD} 4^{+} \mathrm{T}$ cell, and $\mathrm{CD} 69^{+} \mathrm{CD} 4^{+} \mathrm{T}$ cells (CD69: a marker of $\mathrm{T}$ cell activation). The frequency of $\mathrm{CD} 69^{+} \mathrm{CD} 4{ }^{+} \mathrm{T}$ cells was $13.5-$ and 35-fold higher in S@CS(H) immunized mice than in the free spike and control groups in lungs, respectively. Therefore, the results indicated that the increased percentage of $\mathrm{CD} 4^{+} \mathrm{T}$ cells was associated with the magnitude of SARS-CoV-2 spike-specific IgG 

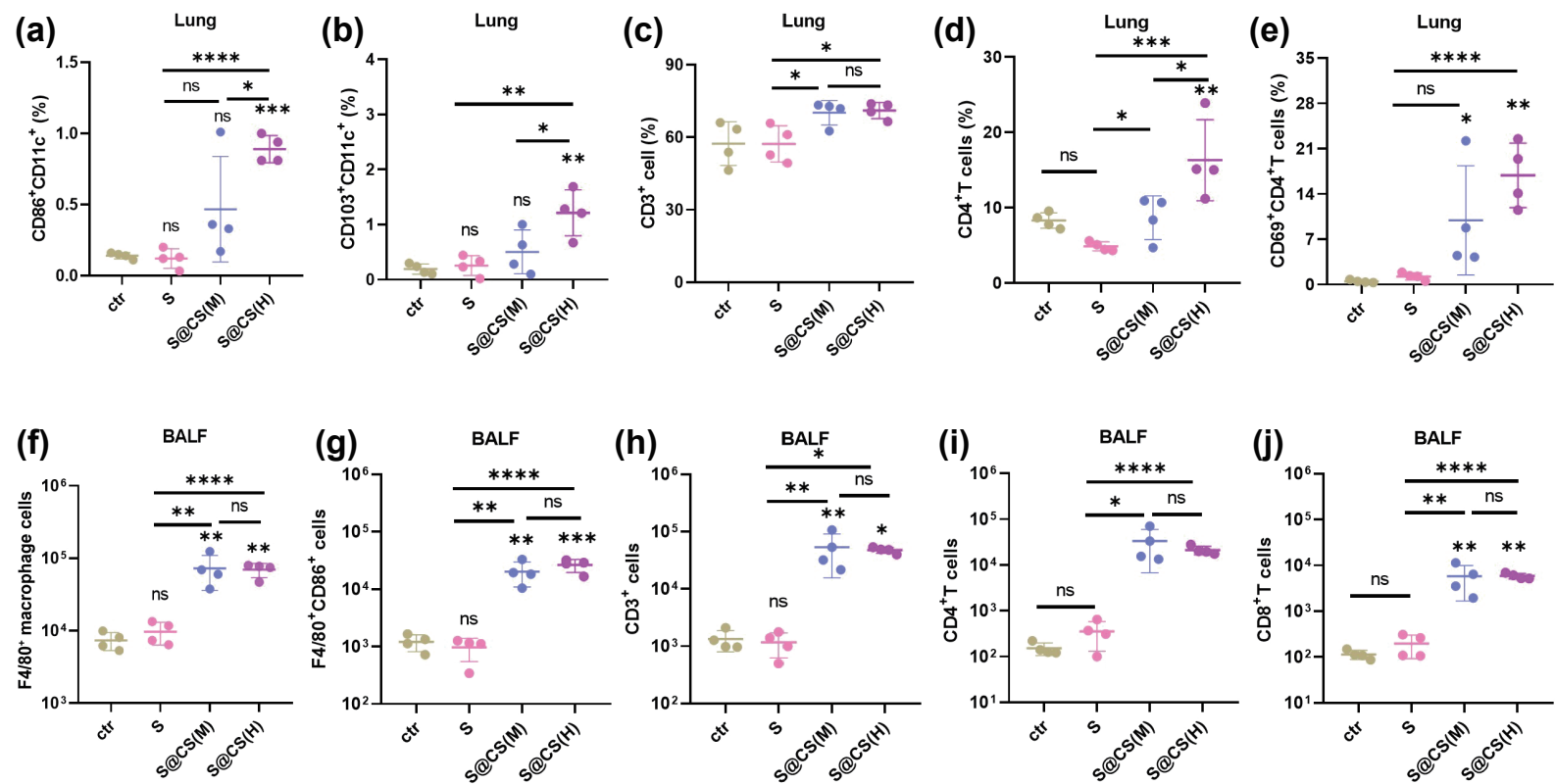

Figure 5 The S@CS nanovaccine potentiated mucosal immunity in BALF and lungs after the third immunization. BALF and lungs were isolated three days following the last immunization and analyzed individually by flow cytometry. (a) and (e) Frequency of immune cells in lungs. (f) and (j) Quantification of immune cells in BALF. The results are presented as cell numbers. All data were presented as the mean $\pm \mathrm{SD}$, and statistical analysis was performed by one-way ANOVA: $* P<0.05, * * P<0.01$, $* * * P<0.001, * * * * P<0.0001$. ns, no significant differences.

and IgA antibody titers (Figs. 2 and 3), which played an essential role in COVID-19 patients and unexposed individuals [46]. Most importantly, the augmented $\mathrm{CD}^{+} \mathrm{T}$ cell response was more important than $\mathrm{CD} 8^{+} \mathrm{T}$ cell response controlling virus infection against SARS-CoV-2 $[34,52]$. In addition, the slightly increased B cells and plasma cells in the $\mathrm{S} @ \mathrm{CS}(\mathrm{H})$ groups were consistent with the enhanced spike-specific antibody responses, which were vital for the production of antibodies and long-term humoral immunity (Figs. S5(b) and S5(c) in the ESM). Taken together, these data showed that the inhalable S@CS nanovaccine might be capable of substantially stimulating the local mucosal immune in BALF and lungs, preventing the virus from initiating infection.

To further characterize whether mucosal immunity belonged to the Th1 type or Th2 type, Th1 cytokines (IFN- $\gamma$ and TNF- $\alpha$ ) and Th2 cytokines (IL-4 and IL-5) in BALF were analyzed by ELISA (Fig. S7 in the ESM). The upregulation of TNF- $\alpha$, IL-4, and IL-5 was consistent with the effective production of IgG1 and IgG2a antibodies, while the expression of IFN- $\gamma$ was not significantly changed. In addition, the expression levels of IL-4 and IL-5 increased more than TNF- $\alpha$ and IFN- $\gamma$, which again indicated strong humoral immunity and balanced cellular immunity.

We verified the biosafety of the S@CS nanovaccine in vivo (Fig. 6). Serum samples were obtained three days after the third immunization, and body weight was recorded on days $0,7,14,21$, and 28. The mouse weight was not significantly altered during immunization (Fig. 6(a)). The serum biochemistry remained in the normal range with nanovaccine inhalation (Figs. 6(b) and 6(c), Fig. S8 in the ESM). In addition, H\&E samples, including the mouse heart, liver, spleen, lungs, and kidneys, were harvested one day after a single immunization. The results suggested that S@CS nanovaccine inhalation did not induce inflammation or organ damage (Fig. 6(d) and Fig. S9 in the ESM) compared to the control group, indicating that the S@CS nanovaccine was biosafe and biocompatible.

\subsection{The inhalable nanovaccine induced comparable} antibody levels compared with intramural injection

To compare the effects of different administration routes on immune responses, we vaccinated three times with S@CS inhalation or intramuscular injection (S@CS (i.m.)) in mice. Serum samples were collected at the indicated times (Fig. 7(a). An equal amount of serum from each individual $(n=9-11)$ in each group was mixed together for ELISA titer assays. As shown in Fig. 7(b), the levels of spike-specific IgG response increased in the S@CS groups. Notably, the inhalation nanovaccine induced a comparable antibody response compared with S@CS (i.m.) treatment. In addition, the body weight (Fig. 7(c)) was at a normal level during immunization, confirming the biosafety of CS. Therefore, based on the SARS-CoV-2 spike-specific antibody response, we shed light on developing the CS-mediated nanovaccine in COVID-19 and speculate on the stimulation of mucosal immunity. Moreover, the strategy satisfied the requirements for large-scale production and immunization anywhere without the need for needles.

\section{Conclusions}

This study developed a noninvasive pulmonary inhalation nanovaccine against SARS-CoV-2 that mimicked the virus entry route and induced local mucosal immunity in the respiratory tract. The CS-mediated inhalable nanovaccine stimulated balanced immunity between humoral and cellular immunity without systemic toxicity, which satisfied the demand for a SARS-CoV-2 vaccine. Additionally, the S@CS nanovaccine induced a comparable antibody immune response against SARS-CoV-2 compared with intramuscular injection. Moreover, the inhalable nanovaccine could reduce the use of needles and is compliant for patients and the public. In addition, CS is relatively inexpensive and available widely, making it suitable for producing a robust vaccine, especially for low-income countries. However, further studies should be carried out in non-human primate to verify the effective protection in COVID-19 with S@CS nanovaccine inhalation. Hence, a CS-mediated noninvasive inhalable nanovaccine strategy is highly translatable as a potential treatment for SARS-CoV-2 and other pulmonary diseases.

\section{Acknowledgements}

This work was supported by the National Key R\&D Program of 

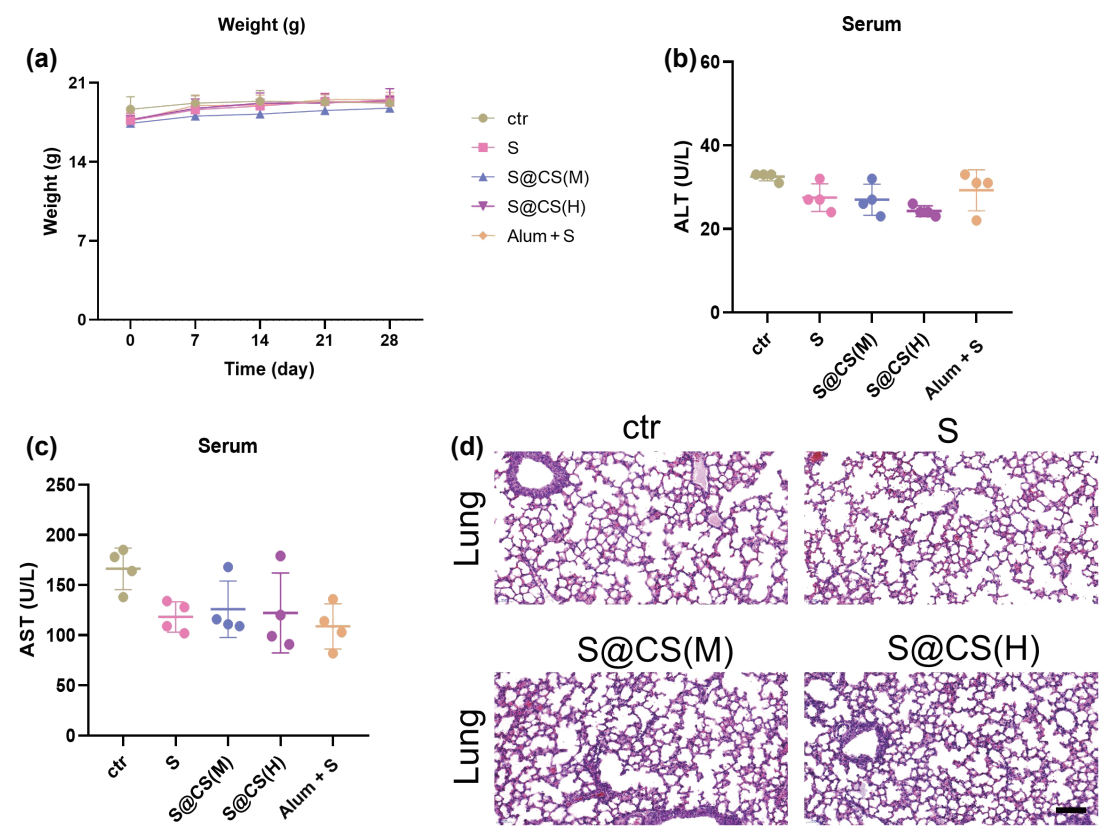

Figure 6 The biosafety of CS-mediated nanovaccine in vivo. (a) Body weight was recorded during immunization. (b) and (c) Quantification of aspartate alanine aminotransferase (ALT) and aspartate aminotransferase (AST). (d) Representative H\&E imaging of lung tissues isolated from mice one day after a single immunization with inhalable nanovaccine. $(n=3$, scale bar: $100 \mu \mathrm{m})$. All data were presented as the mean \pm SD, and statistical analysis was performed by one-way ANOVA: $* P<$ $0.05, * * P<0.01, * * * P<0.001, * * * * P<0.0001$. ns, no significant differences.

(a)
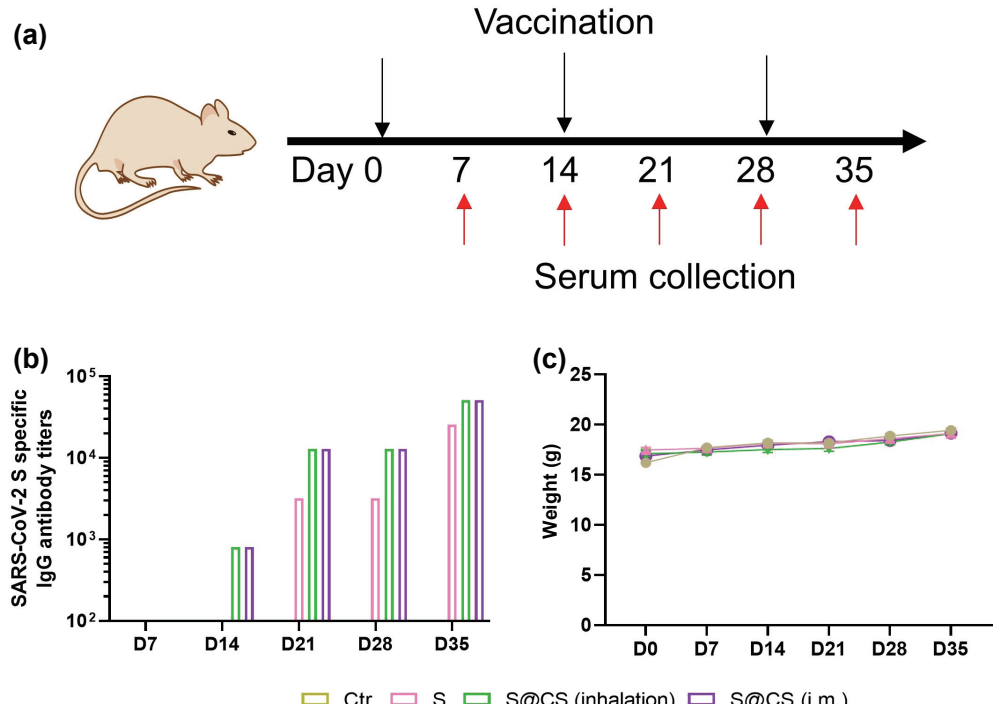

Figure 7 The effects of different administration routes on immune responses in BALB/c mice. (a) Schedule of immunization and serum collection in BALB/c mice $(n$ = 9-11). Mice were separated into four groups. Three groups underwent inhalation, including the PBS group, S and S@CS (inhalation). S@CS was also intramuscularly injected as a control (S@CS (i.m.)). (b) SARS-CoV-2 spike-specific IgG antibody titers in sera the indicated times. (c) Body weight was recorded during immunization. All data were presented as the mean $\pm \mathrm{SD}$, and statistical analysis was performed by one-way ANOVA: $* P<0.05, * * P<0.01, * * * P<0.001, * * * * P<$ 0.0001. ns, no significant differences.

China (Nos. 2019YFA0904200 and 2018YFA0507600), Tsinghua University Spring Breeze Fund (No. 2020Z99CFY042), and the National Natural Science Foundation of China (No. 92053108). Applications for Chinese patents related to chitosan-mediated inhalable nanovaccine has been submitted (Patent No. 202111130951.7). We thank Prof. Yanan Du (Tsinghua University) for kind support with the microsprayer aerosolizers and laryngoscope in the early stage of this work and Dr. Rui Kuai (Tsinghua University) for kind support with laryngoscopy in this research.

Electronic Supplementary Material: Supplementary material (the immune activation of CS-mediated nanovacccine on BMDCs, cell viability, immune responses in lungs and BALF, serum chemistry and H\&E histopathological analysis.) is available in the online version of this article at https://doi.org/10.1007/s12274-0214012-9.

\section{References}

[1] Davies, N. G.; Abbott, S.; Barnard, R. C.; Jarvis, C. I.; Kucharski, A. J.; Munday, J. D.; Pearson, C. A. B.; Russell, T. W.; Tully, D. C.; Washburne, A. D. et al. Estimated transmissibility and impact of SARS-CoV-2 lineage B.1.1.7 in England. Science 2021, 372, eabg3055.

[2] Nonaka, C. K. V.; Franco, M. M.; Gräf, T.; de Lorenzo Barcia, C. A.; de Ávila Mendonça, R. N.; de Sousa, K. A. F.; Neiva, L. M. C.; Fosenca, V.; Mendes, A. V. A.; de Aguiar, R. S. et al. Genomic evidence of SARS-CoV-2 reinfection involving E484K spike mutation, brazil. Emerg. Infect. Dis. 2021, 27, 1522-1524.

[3] Tegally, H.; Wilkinson, E.; Giovanetti, M.; Iranzadeh, A.; Fonseca, V.; Giandhari, J.; Doolabh, D.; Pillay, S.; San, E. J.; Msomi, N. et al. 
Detection of a SARS-CoV-2 variant of concern in South Africa. Nature 2021, 592, 438-443.

[4] Liu, Y.; Liu, J. Y.; Xia, H. J.; Zhang, X. W.; Fontes-Garfias, C. R.; Swanson, K. A.; Cai, H.; Sarkar, R.; Chen, W.; Cutler, M. et al. Neutralizing activity of BNT162b2-elicited serum. N. Engl. J. Med. 2021, 384, 1466-1468.

[5] Chen, R. E.; Zhang, X. W.; Case, J. B.; Winkler, E. S.; Liu, Y.; VanBlargan, L. A.; Liu, J. Y.; Errico, J. M.; Xie, X. P.; Suryadevara, $\mathrm{N}$. et al. Resistance of SARS-CoV-2 variants to neutralization by monoclonal and serum-derived polyclonal antibodies. Nat. Med. 2021, 27, 717-726.

[6] Wang, P. F.; Nair, M. S.; Liu, L. H.; Iketani, S.; Luo, Y.; Guo, Y. C.; Wang, M.; Yu, J.; Zhang, B. S.; Kwong, P. D. et al. Antibody resistance of SARS-CoV-2 variants B.1.351 and B.1.1.7. Nature 2021, 593, 130-135.

[7] Wang, Z. J.; Schmidt, F.; Weisblum, Y.; Muecksch, F.; Barnes, C. O.; Finkin, S.; Schaefer-Babajew, D.; Cipolla, M.; Gaebler, C.; Lieberman, J. A. et al. mRNA vaccine-elicited antibodies to SARSCoV-2 and circulating variants. Nature 2021, 592, 616-622.

[8] Letko, M.; Marzi, A.; Munster, V. Functional assessment of cell entry and receptor usage for SARS-CoV-2 and other lineage B betacoronaviruses. Nat. Microbiol. 2020, 5, 562-569.

[9] Zhou, P.; Yang, X. L.; Wang, X. G.; Hu, B.; Zhang, L.; Zhang, W.; Si, H. R.; Zhu, Y.; Li, B.; Huang, C. L. et al. A pneumonia outbreak associated with a new coronavirus of probable bat origin. Nature 2020, 579, 270-273.

[10] Acter, T.; Uddin, N.; Das, J.; Akhter, A.; Choudhury, T. R.; Kim, S. Evolution of severe acute respiratory syndrome coronavirus 2 (SARS-CoV-2) as coronavirus disease 2019 (COVID-19) pandemic: A global health emergency. Sci. Total Environ. 2020, 730, 138996.

[11] Li, H. J.; Wang, Y. Y.; Ji, M. Y.; Pei, F. Y.; Zhao, Q. Q.; Zhou, Y. Y.; Hong, Y. T.; Han, S. Y.; Wang, J.; Wang, Q. X. et al. Transmission routes analysis of SARS-CoV-2: A systematic review and case report. Front. Cell Dev. Biol. 2020, 8, 618.

[12] Hassan, A. O.; Feldmann, F.; Zhao, H. Y.; Curiel, D. T.; Okumura, A.; Tang-Huau, T. L.; Case, J. B.; Meade-White, K.; Callison, J.; Chen, R. E. et al. A single intranasal dose of chimpanzee adenovirusvectored vaccine protects against SARS-CoV-2 infection in rhesus macaques. Cell Rep. Med. 2021, 2, 100230.

[13] Polack, F. P.; Thomas, S. J.; Kitchin, N.; Absalon, J.; Gurtman, A.; Lockhart, S.; Perez, J. L.; Marc, G. P.; Moreira, E. D.; Zerbini, C. et al. Safety and efficacy of the BNT162b2 mRNA covid-19 vaccine. N. Engl. J. Med. 2020, 383, 2603-2615.

[14] Baden, L. R.; El Sahly, H. M.; Essink, B.; Kotloff, K.; Frey, S.; Novak, R.; Diemert, D.; Spector, S. A.; Rouphael, N.; Creech, C. B. et al. Efficacy and safety of the mRNA-1273 SARS-CoV-2 vaccine. N. Engl. J. Med. 2021, 384, 403-416.

[15] Sadoff, J.; Le Gars, M.; Shukarev, G.; Heerwegh, D.; Truyers, C.; de Groot, A. M.; Stoop, J.; Tete, S.; Van Damme, W.; Leroux-Roels, I. et al. Interim results of a phase 1-2a trial of Ad26.COV2.S Covid-19 vaccine. N. Engl. J. Med. 2021, 384, 1824-1835.

[16] Jin, Q. T.; Zhu, W. J.; Zhu, J. F.; Zhu, J. J.; Shen, J. J.; Liu, Z.; Yang, Y.; Chen, Q. Nanoparticle-mediated delivery of inhaled immunotherapeutics for treating lung metastasis. Adv. Mater. 2021, 33,2007557

[17] Zhang, H.; Zhu, W. J.; Jin, Q. T.; Pan, F.; Zhu, J. F.; Liu, Y. B.; Chen, L. F.; Shen, J. J.; Yang, Y.; Chen, Q. et al. Inhalable nanocatchers for SARS-CoV-2 inhibition. Proc. Natl. Acad. Sci. USA 2021, 118, e2102957118.

[18] Zheng, B.; Peng, W. C.; Guo, M. M.; Huang, M. Q.; Gu, Y. X.; Wang, T.; Ni, G. J.; Ming, D. Inhalable nanovaccine with biomimetic coronavirus structure to trigger mucosal immunity of respiratory tract against COVID-19. Chem. Eng. J. 2021, 418, 129392.

[19] Lycke, N. Recent progress in mucosal vaccine development: Potential and limitations. Nat. Rev. Immunol. 2012, 12, 592-605.

[20] Gerada, C.; Campbell, T. M.; Kennedy, J. J.; McSharry, B. P.; Steain, M.; Slobedman, B.; Abendroth, A. Manipulation of the innate immune response by varicella zoster virus. Front. Immunol. 2020, 11, 1.

[21] Lavelle, E. C.; Ward, R. W. Mucosal vaccines-fortifying the frontiers. Nat. Rev. Immunol., in press, https/: doi.org/10.1038/s41577-021-00583-2.

[22] Carroll, E. C.; Jin, L.; Mori, A.; Muñoz-Wolf, N.; Oleszycka, E.; Moran, H. B. T.; Mansouri, S.; McEntee, C. P.; Lambe, E.; Agger, E. M. et al. The vaccine adjuvant chitosan promotes cellular immunity via DNA sensor cGAS-STING-dependent induction of type i interferons. Immunity 2016, 44, 597-608.

[23] Li, W. H.; Li, Y. M. Chemical strategies to boost cancer vaccines Chem. Rev. 2020, 120, 11420-11478.

[24] Wu, J. J.; Zhao, L.; Hu, H. G.; Li, W. H.; Li, Y. M. Agonists and inhibitors of the sting pathway: Potential agents for immunotherapy. Med. Res. Rev. 2020, 40, 1117-1141.

[25] Bedford, J. G.; Caminschi, I.; Wakim, L. M. Intranasal delivery of a chitosan-hydrogel vaccine generates nasal tissue resident memory $\mathrm{CD}^{+} \mathrm{T}$ cells that are protective against influenza virus infection. Vaccines (Basel) 2020, 8, 572.

[26] Tatlow, D.; Tatlow, C.; Tatlow, S.; Tatlow, S. A novel concept for treatment and vaccination against Covid-19 with an inhaled chitosancoated DNA vaccine encoding a secreted spike protein portion. Clin. Exp. Pharmacol. Physiol. 2020, 47, 1874-1878.

[27] Jearanaiwitayakul, T.; Seesen, M.; Chawengkirttikul, R.; Limthongkul, J.; Apichirapokey, S.; Sapsutthipas, S.; Phumiamorn, S.; Sunintaboon, P.; Ubol, S. Intranasal administration of RBD nanoparticles confers induction of mucosal and systemic immunity against SARS-CoV-2. Vaccines (Basel) 2021, 9, 768.

[28] Zihni, C.; Mills, C.; Matter, K.; Balda, M. S. Tight junctions: From simple barriers to multifunctional molecular gates. Nat. Rev. Mol. Cell Biol. 2016, 17, 564-580.

[29] Alitongbieke, G.; Li, X. M.; Wu, Q. C.; Lin, Z. C.; Huang, J. F.; Xue, Y.; Liu, J. N.; Lin, J. M.; Pan, T.; Chen, Y. X. et al. Effect of $\beta$ chitosan on the binding interaction between SARS-CoV-2 S-RBD and ACE2. bioRxiv 2020.07.31.229781, 2020. Available at https://doi.org/10.1101/2020.07.31.229781.

[30] Milewska, A.; Chi, Y.; Szczepanski, A.; Barreto-Duran, E.; Dabrowska, A.; Botwina, P.; Obloza, M.; Liu, K.; Liu, D.; Guo, X. L. et al. HTCC as a polymeric inhibitor of SARS-CoV-2 and MERSCoV. J. Virol. 2021, 95, e01622-20.

[31] Sharma, N.; Modak, C.; Singh, P. K.; Kumar, R.; Khatri, D.; Singh, S. B. Underscoring the immense potential of chitosan in fighting a wide spectrum of viruses: A plausible molecule against SARS-CoV2? Int. J. Biol. Macromol. 2021, 179, 33-44.

[32] Giurgea, L. T.; Han, A.; Memoli, M. J. Universal coronavirus vaccines: The time to start is now. npj Vaccines 2020, 5, 43-45.

[33] Wang, J.; Chin, D.; Poon, C.; Mancino, V.; Pham, J.; Li, H.; Ho, P Y.; Hallows, K. R.; Chung, E. J. Oral delivery of metformin by chitosan nanoparticles for polycystic kidney disease. J. Control. Release 2021, 329, 1198-1209.

[34] Sette, A.; Crotty, S. Adaptive immunity to SARS-CoV-2 and COVID-19. Cell 2021, 184, 861-880.

[35] Moderbacher, C R.; Ramirez, S. I.; Dan, J. M.; Grifoni, A.; Hastie, K. M.; Weiskopf, D.; Belanger, S.; Abbott, R. K.; Kim, C.; Choi, J. et al. Antigen-specific adaptive immunity to SARS-CoV-2 in acute COVID-19 and associations with age and disease severity. Cell 2020, 183, 996-1012.

[36] Cai, H.; Sun, Z. Y.; Chen, M. S.; Zhao, Y. F.; Kunz, H.; Li, Y. M. Synthetic multivalent glycopeptide-lipopeptide antitumor vaccines: Impact of the cluster effect on the killing of tumor cells. Angew. Chem., Int. Ed. 2014, 53, 1699-1703.

[37] Wu, J. J.; Li, W. H.; Chen, P. G.; Zhang, B. D.; Hu, H. G.; Li, Q. Q.; Zhao, L.; Chen, Y. X.; Zhao, Y. F.; Li, Y. M. Targeting STING with cyclic di-GMP greatly augmented immune responses of glycopeptide cancer vaccines. Chem. Commun. 2018, 54, 9655-9658.

[38] Wu, J. J.; Zhao, L.; Han, B. B.; Hu, H. G.; Zhang, B. D.; Li, W. H.; Chen, Y. X.; Li, Y. M. A novel STING agonist for cancer immunotherapy and a SARS-CoV-2 vaccine adjuvant. Chem. Commun. 2021, 57, 504-507.

[39] Seregin, S. S.; Appledorn, D. M.; McBride, A. J.; Schuldt, N. J.; Aldhamen, Y. A.; Voss, T.; Wei, J. P.; Bujold, M.; Nance, W.; Godbehere, S. et al. Transient pretreatment with glucocorticoid ablates innate toxicity of systemically delivered adenoviral vectors without reducing efficacy. Mol. Ther. 2009, 17, 685-696. 
[40] Cai, H.; Huang, Z. H.; Shi, L.; Sun, Z. Y.; Zhao, Y. F.; Kunz, H.; Li, Y. M. Variation of the glycosylation pattern in MUC1 glycopeptide BSA vaccines and its influence on the immune response. Angew. Chem., Int. Ed. 2012, 51, 1719-1723.

[41] Huang, Z. H.; Shi, L.; Ma, J. W.; Sun, Z. Y.; Cai, H.; Chen, Y. X.; Zhao, Y. F.; Li, Y. M. a totally synthetic, self-assembling, adjuvantfree MUC1 glycopeptide vaccine for cancer therapy. J. Am. Chem. Soc. 2012, 134, 8730-8733.

[42] See, R. H.; Zakhartchouk, A. N.; Petric, M.; Lawrence, D. J.; Mok, C. P. Y.; Hogan, R. J.; Rowe, T.; Zitzow, L. A.; Karunakaran, K. P.; Hitt, M. M. et al. Comparative evaluation of two severe acute respiratory syndrome (SARS) vaccine candidates in mice challenged with SARS coronavirus. J. Gen. Virol. 2006, 87, 641-650.

[43] Sterlin, D.; Mathian, A.; Miyara, M.; Mohr, A.; Anna, F.; Claër, L.; Quentric, P.; Fadlallah, J.; Devilliers, H.; Ghillani, P. et al. IgA dominates the early neutralizing antibody response to SARS-CoV-2. Sci. Transl. Med. 2021, 13, eabd2223.

[44] Cervia, C.; Nilsson, J.; Zurbuchen, Y.; Valaperti, A.; Schreiner, J.; Wolfensberger, A.; Raeber, M. E.; Adamo, S.; Weigang, S.; Emmenegger, M. et al. Systemic and mucosal antibody responses specific to SARS-CoV-2 during mild versus severe COVID-19. J. Allergy Clin. Immunol. 2021, 147, 545-557.e9.

[45] Shao, Y.; Sun, Z. Y.; Wang, Y. J.; Zhang, B. D.; Liu, D. S.; Li, Y. M. Designable immune therapeutical vaccine system based on DNA supramolecular hydrogels. ACS Appl. Mater. Interfaces 2018, 10, 9310-9314.

[46] Grifoni, A.; Weiskopf, D.; Ramirez, S. I.; Mateus, J.; Dan, J. M.; Moderbacher, C. R.; Rawlings, S. A.; Sutherland, A.; Premkumar,
L.; Jadi, R. S. et al. Targets of T cell responses to SARS-CoV-2 coronavirus in humans with COVID-19 disease and unexposed individuals. Cell 2020, 181, 1489-1501.

[47] Le Bert, N.; Tan, A. T.; Kunasegaran, K.; Tham, C. Y. L.; Hafezi, M.; Chia, A.; Chng, M. H. Y.; Lin, M. Y.; Tan, N.; Linster, M. et al. SARS-CoV-2-specific T cell immunity in cases of COVID-19 and SARS, and uninfected controls. Nature 2020, 584, 457-462.

[48] Sekine, T.; Perez-Potti, A.; Rivera-Ballesteros, O.; Strålin, K.; Gorin, J. B.; Olsson, A.; Llewellyn-Lacey, S.; Kamal, H.; Bogdanovic, G.; Muschiol, S. et al. Robust $\mathrm{T}$ cell immunity in convalescent individuals with asymptomatic or mild COVID-19. Cell 2020, 183, $158-168$

[49] Pušnik, J.; Richter, E.; Schulte, B.; Dolscheid-Pommerich, R.; Bode, C.; Putensen, C.; Hartmann, G.; Alter, G.; Streeck, H. Memory B cells targeting SARS-CoV-2 spike protein and their dependence on $\mathrm{CD}^{+} \mathrm{T}$ cell help. Cell Rep. 2021, 35, 109320.

[50] Yuan, Z. X.; Sun, X.; Gong, T.; Ding, H.; Fu, Y.; Zhang, Z. R. Randomly $50 \% \mathrm{~N}$-acetylated low molecular weight chitosan as a novel renal targeting carrier. J. Drug Target. 2007, 15, 269-278.

[51] Liu, Y.; Crowe, W. N.; Wang, L. L.; Lu, Y.; Petty, W. J.; Habib, A A.; Zhao, D. W. An inhalable nanoparticulate STING agonis synergizes with radiotherapy to confer long-term control of lung metastases. Nat. Commun. 2019, 10, 5108.

[52] Painter, M. M.; Mathew, D.; Goel, R. R.; Apostolidis, S. A.; Pattekar, A.; Kuthuru, O.; Baxter, A. E.; Herati, R. S.; Oldridge, D. A.; Gouma, S. et al. Rapid induction of antigen-specific $\mathrm{CD} 4^{+} \mathrm{T}$ cells is associated with coordinated humoral and cellular immunity to SARSCoV-2 mRNA vaccination. Immunity 2021, 54, 2133-2142. 